\title{
Role of urine osmolality as a predictor of the effectiveness of combined imipramine and desmopressin in the treatment of monosymptomatic nocturnal enuresis
}

\author{
Kwon Soo Lee', Jun Bo Chang ${ }^{1}$, Jae Yoon Jang ${ }^{1}$, Young Hwii Ko', Yong Hoon Park ${ }^{2}$, Phil Hyun Song ${ }^{1}$ \\ Departments of ${ }^{1}$ Urology and ${ }^{2}$ Pediatrics, College of Medicine, Yeungnam University, Daegu, Korea
}

Background: We examined the usefulness of urine osmolality, as a predictive factor in the treatment of monosymptomatic nocturnal enuresis (NE) with combination therapy of imipramine and desmopressin.

Methods: From May 2014 to April 2015, 59 monosymptomatic NE patients participated in this study. Early morning urine osmolality was measured at 1 week and 1 day before combination therapy of imipramine and desmopressin, and at 1 week and 2 weeks after therapy. The response to combination therapy was evaluated at 3 months after treatment. The mean period of combination therapy was $6.4 \pm 4.2$ weeks. Therapeutic response was classified as complete (0-1 wet night/week), partial (over $50 \%$ reduction of night) and non-responders (less than $50 \%$ reduction of night). Results: The cumulative rate of the complete and partial responders was $76.3 \%$. Among the 3 groups, the statistically lowest value of pre-treatment urine osmolality was observed in the complete responder group $(p<0.001)$. Urine osmolality increased in all groups after treatment, however, statistically the greatest difference between pre and post-treatment urine osmolality was observed in the complete responder group $(p=0.024)$. No serious side effects were observed. Conclusion: Early morning urine osmolality and change of urine osmolality between pre and post-treatment have predictive values in the response to combined imipramine and desmopressin for treatment of monosymptomatic NE.

Keywords: Enuresis; Urine; Osmolality

\section{INTRODUCTION}

Nocturnal enuresis (NE) refers to cases involving children aged 5 years or older who are not able to control the urinary bladder during night sleeping more than twice a month due to involuntary urine leakage [1]. Primary NE refers to cases involving children who persistently cannot control the bladder after birth. Secondary NE refers to cases in which the bladder control remains unproblematic until a specific time point but symptoms of NE recur after approximately 6 months to one year. NE can also be classified according to polysymptomatic $\mathrm{NE}$ where there is a concurrent presence of daytime oliguria,

Received: October 29, 2015, Revised: November 10, 2015 Accepted: November 10, 2015

Corresponding Author: Phil Hyun Song, Department of Urology, College of Medicine, Yeungnam University, 170 Hyunchung-ro, Nam-gu, Daegu 42415, Korea

Tel: +82-53-620-3693, Fax: +82-53-627-5535

E-mail: sph04@hanmail.net urgency, urinary incontinence or daytime enuresis and monosymptomatic $\mathrm{NE}$ where there is a single presence of $\mathrm{NE}$ [2]. The prevalence of $\mathrm{NE}$ is estimated at approximately $15 \%$ in children aged 5 years or so. After the age of 5 years, the annual rate of spontaneous recovery is estimated at $15 \%$. Accordingly it is recovered spontaneously in most children aged 10 years or older [3]. In recent years, however, NE has been reported to have a detrimental effect on children's mental health and to cause a lack of confidence [4]. In addition, with the improved quality of life and the decreased number of children, efforts in treatment of $\mathrm{NE}$ have also increased.

To date in Korea, a substantial number of studies have reported that drug treatments with a single or concomitant use of imipramine and desmopressin are effective in patients with $\mathrm{NE}[5,6$. Their treatment effects are assumed to be good from an overall perspective. However, opinions regarding the treatment effects of these drugs are controversial. Age, a family history, and urine osmolality may also affect the treatment response to drugs [7]. Of patients who received concomitant 
medication with imipramine and desmopressin, $20 \%-40 \%$ are refractory to the treatment. Accordingly, indicators predicting the treatment effects would greatly contribute to the treatment of NE.

Given the above described background, we analyzed the treatment response to concomitant medication with imipramine and desmopressin in children with monosymptomatic $\mathrm{NE}$ for which other organic causes could be ruled out through a screening test. We also examined the question of whether urine osmolality has a prognostic value for treatment response in an outpatient setting.

\section{MATERIALS AND METHODS}

\section{Patients}

Of children who visited the outpatient clinic of the department of pediatrics and urology at our medical institution with a chief complaint of NE during a period ranging from May 2014 to April 2015, 59 patients with monosymptomatic NE for which organic causes could be ruled out through a screening test and who did not undergo drug treatments for $\mathrm{NE}$ during a recent 2-month period were included in the current study. As a screening test for NE, meticulous history taking, physical examination, urinalysis, and urine culture were performed for all cases. Through meticulous history taking, the pattern of $\mathrm{NE}$, the presence of oliguria or urgency, the age at which bladder control was achieved, a family history, and a past history of urinary tract infection were examined. The dorsal region and neurological deficits were evaluated for abnormality during physical examination to determine whether there was a concurrent presence of anatomical abnormalities and neurological deficits. Radiography or uroflowmetry was performed in cases in which a urinary system abnormality or neurological deficits were suspected on a screening test. Cases in which abnormal findings were observed and those of severe constipation were excluded from the current analysis.

The mean age of 59 subjects, consisting of 38 boys and 21 girls, was 8.3 years (5-16). There were 45 cases of primary $\mathrm{NE}$ and 14 cases of secondary NE (Table 1). The mean period of combination therapy was $6.4 \pm 4.2$ weeks.

\section{Methods}

As the treatment methods, in addition to water restriction prior to sleeping, supportive treatment included a voiding diary and drug treatments. Water intake was restricted from 2 hours before sleeping, and voluntary urination before sleeping was recommended. As supportive treatment, the motive of treatment was explained to the subjects. Voluntary recording in the voiding diary was recommended during treatment. Then, at an outpatient visit, children were encouraged through an analysis of this diary. In 59 subjects, urine osmolality (baseline level) was measured from the first urine in the morning twice one week and one day before the administration of drugs. For treatment of NE, imipramine $25 \mathrm{mg}$ and desmopressin $0.2 \mathrm{mg}$ were administered orally before sleeping. During weeks 1 and 2 following treatment, urine osmolality was measured for the first urine in the morning. Three months later, an analysis was performed for the treatment effects and side effects. In cases in which there were no treatment responses during week 2 following the administration of drugs, the dose of desmopressin was increased to $0.4 \mathrm{mg}$. For determination of treatment responses to drugs, children were classified according to 3 groups: the complete responder group where the weekly incidence of $\mathrm{NE}$ was decreased to $0-1$ times, the partial responder group where the weekly incidence of $\mathrm{NE}$ was lower by $50 \%$ as compared with baseline, and the non-res-

Table 1. Sex and type of monosymptomatic nocturnal enuresis distribution according to age

\begin{tabular}{cccccccc}
\hline \multirow{2}{*}{ Age $(\mathrm{yr})$} & \multicolumn{7}{c}{ No. of Patient (\%) } \\
\cline { 2 - 4 } \cline { 6 - 8 } & Male & Female & Total & & Primary enuresis & Secondary enuresis & Total \\
\hline $5-6$ & $8(13.6)$ & $4(6.8)$ & $12(20.3)$ & & $11(18.6)$ & $1(1.7)$ & $12(20.3)$ \\
$7-8$ & $15(25.4)$ & $8(13.6)$ & $23(39.0)$ & & $19(32.2)$ & $4(6.8)$ & $23(39.0)$ \\
$9-10$ & $9(15.3)$ & $6(10.2)$ & $15(25.4)$ & & $11(18.6)$ & $4(6.8)$ & $15(25.4)$ \\
$11-12$ & $4(6.8)$ & $3(5.1)$ & $7(11.9)$ & & $3(5.1)$ & $4(6.8)$ & $7(11.9)$ \\
$13-14$ & $1(1.7)$ & 0 & $1(1.7)$ & & 0 & $1(1.7)$ & $1(1.7)$ \\
$15-16$ & $1(1.7)$ & 0 & $1(1.7)$ & & $1(1.7)$ & 0 & $1(1.7)$ \\
Total & $38(64.4)$ & $21(35.6)$ & $59(100)$ & & $45(76.3)$ & $14(23.7)$ & $59(100)$ \\
\hline
\end{tabular}


ponder group where there was no change in the weekly incidence of $\mathrm{NE}$ or it was higher than 50\%. In each group, changes in urine osmolality were compared between before and after treatment. The complete responder group and the partial responder group were considered to show a treatment effect. In cases involving a persistent complete response for more than 4 weeks, the treatment was discontinued. Subjects who had completed the treatment underwent follow-up observation for examination of recurrence. Urine osmolality was measured using a freezing point osmometer, Advanced Osmometer 2020 (Advanced Instruments INC., USA). A $10 \mu \mathrm{L}$ sample of urine was used for the test.

\section{Statistical analysis of the treatment outcomes}

Statistical analysis of the treatment outcomes was performed using Chi-square test and ANOVA, using SPSS $17.0 \mathrm{~K}$. A value of $p<0.05$ was considered statistically significant.

\section{RESULTS}

Of 59 patients in this study, there were 29 patients (49.2\%) of complete response, 16 patients (27.1\%) of partial response and 14 patients (23.7\%) of no response. That is, the treatment effects were observed in $76.3 \%$ of patients. Mean period of treatment response, defined as the period in which the incidence of NE was decreased by more than $50 \%$ in the complete responder group and the partial responder group, was $2.8 \pm 2.3$ weeks. The mean weekly incidence of $\mathrm{NE}$ prior to treatment was $6.1 \pm 1.8$ times in the complete responder group, $6.6 \pm 1.5$ times in the partial responder group, and $6.5 \pm 2.0$ times in the non-responder group. These differences did not reach statistical significance $(p=0.685)$. Following treatment, however, the mean weekly incidence of $\mathrm{NE}$ was $0.4 \pm 0.5$ times, $2.3 \pm 0.5$ times, and $5.7 \pm 1.4$ times in the corresponding order.

Following a comparison of the treatment effect between the primary $\mathrm{NE}$ and the secondary $\mathrm{NE}$, the treatment effect was observed in $75.5 \%$ of patients of primary NE (42.2\% of complete responses and 33.3\% of partial responses) and $78.6 \%$ of patients of secondary NE. These results indicate that the treatment effect was relatively good. However, there was no statistical significance in this series $(p>0.05)$ (Table 2).

Prior to treatment, urine osmolality was measured as 461.2 $\pm 192.7,596.9 \pm 197.3$, and $773.5 \pm 235.8 \mathrm{mOsm} / \mathrm{kg}$ in the complete, partial, and non-responder group, respectively. These differences reached statistical significance $(p<0.001)$. Compared with before treatment, urine osmolality was increased in each group following treatment. In the complete responder group, however, it was increased from 461.2 $\pm 192.7 \mathrm{mOsm} /$ $\mathrm{kg}$ to $591.2 \pm 159.8 \mathrm{mOsm} / \mathrm{kg}$. These results indicate that the incremental degree of urine osmolality following treatment was the highest in the complete responder group and this reached statistical significance $(p=0.024)$ (Table 3).

Of 29 cases in which treatment was completed due to a complete response, the number of cases of recurrence due to the discontinued administration of drugs was $4(13.8 \%)$ within one month, 1 (3.4\%) within 2 months, 2 (6.9\%) within 3 months, and 3 (10.3\%) after 3 months. That is, the overall rate of recurrence was $34.5 \%$ (10 cases). A comparison of changes in urine osmolality between the recurred cases and other cases in the complete responder group showed no statistical significance.

As the side effects occurring following concomitant use of imipramine and desmopressin, there were 5 cases (8.5\%) of decreased appetite, one case (1.7\%) of xerostomia and one case (1.7\%) of abdominal pain. That is side effects occurred in $11.9 \%$ of subjects. However, these temporary occurrences

Table 2. Response to medical treatment in primary and secondary monosymptomatic nocturnal enuretic patients

\begin{tabular}{lccc}
\hline \multirow{2}{*}{ Responder } & \multicolumn{3}{c}{ No. of patient $(\%)$} \\
\cline { 2 - 4 } & Primary $(\mathrm{n}=45)$ & Secondary $(\mathrm{n}=14)$ & $p$-value \\
\hline Complete $(\mathrm{n}=29)$ & $19(42.2)$ & $10(71.5)$ & 0.056 \\
Partial $(\mathrm{n}=16)$ & $15(33.3)$ & $1(7.1)$ & 0.084 \\
None $(\mathrm{n}=14)$ & $11(24.5)$ & $3(21.4)$ & 0.564 \\
\hline
\end{tabular}

Data were evaluated using Chi-square test.

Table 3. Change of urine osmolality between pre-treatment and post-treatment

\begin{tabular}{lccc}
\hline Responder & $\begin{array}{c}\text { Pre-treatment } \\
(\mathrm{mOsm} / \mathrm{kg})\end{array}$ & $\begin{array}{c}\text { Post-treatment } \\
(\mathrm{mOsm} / \mathrm{kg})\end{array}$ & $p$-value \\
\hline Complete & $461.2 \pm 192.7$ & $591.2 \pm 159.8$ & \\
Partial & $596.9 \pm 197.3$ & $652.9 \pm 164.8$ & 0.024 \\
None & $773.5 \pm 235.8$ & $833.0 \pm 199.2$ & \\
${ }^{a} p$-value & $<0.001$ & $<0.001$ & \\
\hline
\end{tabular}

Values are presented as mean \pm standard deviation.

Data were evaluated using ANOVA.

a) $p$-value means that there were statistical significance in urine osmolality at pre-treatment and post-treatment among 3 responder groups. 
were of mild severity and then improved spontaneously. The severity of these side effects was not so great as to discontinue the administration of drugs.

\section{DISCUSSION}

Historically, drug treatments have long been administered for NE with frequent use of antidepressant, anticholinergics and antidiuretics in recent years. Drug treatments are administered advantageous due to the advantage of easy use and relatively rapid effect. However, there are still limitations, including side effects and a relatively higher recurrence rate.

Imipramine, a tricyclic antidepressant, was first used for children with NE by MacLean during the 1960s. Since then, it has been used the most frequently [8]. Its mode of action is still unknown. However, it is known to stimulate the central nervous system, lessen the depth of rapid eye movement sleep, enhance awakening from sleep and thereby increases the sensitivity to voiding desire. Due to the direct anti-cholinergic effects on the urinary bladder, it increases the volume of the urinary bladder. Due to the excitation on the sympathetic nervous system, it increases the absorption of water and electrolytes and promotes the removal of urea in the collecting duct. It is also known to increase urea elimination leading to the decreased excretion of urine osmolute [9]. Although rare, side effects including sleeping disorder, gastrointestinal disturbances, seizure, anxiety, and syncope have been reported. Therefore use in children before the age of 5 years is not recommended [2].

Desmopressin is a synthetic polypeptide with a structure similar to that of arginine vasopressin, an anti-diuretic hormone released from the posterior lobe of the pituitary gland. It has mainly been used in treatment of patients with diabetes inspidus. According to recent studies, it is a key pathophysiologic mechanism of $\mathrm{NE}$ in which the release of anti-diuretic hormones is not increased during sleeping in patients with $\mathrm{NE}$, unlike normal healthy people. Based on these reports, it has been applauded as a new drug with a prompt action and a lower incidence of side effects [10]. Desmopressin has few side effects. However, transient headache, nausea, face blushing, and mild abdominal pain may occur [10].

Concomitant medication of imipramine with desmopressin has been attempted based on the difference in the mode of action between the 2 drugs. According to Chung and Kim, on month 3 , the treatment effect was observed in $100 \%$ of patients in the imipramine+desmopressin group and $78.2 \%$ of those in the imipramine monotherapy group. These authors therefore noted that concomitant medication of imipramine with desmopressin showed an excellent degree of efficacy as compared with a monotherapy with imipramine [11]. According to Rienberg and Vaughn, in 15 patients who were refractory to a single use of imipramine or oral desmopressin, the rate of treatment success was $73 \%$ following concomitant medication of imipramine with desmopressin [12]. Possible side effects due to concomitant medication include decreased appetite, xerostomia, sleep disorder, and mild abdominal pain [6]. In our series, based on criteria that both the complete responder group and the partial responder group were considered to have a treatment effect, the treatment effect was found in $76.3 \%$ of patients. Side effects included 5 cases (8.5\%) of decreased appetite, one case (1.7\%) of xerostomia and one case (1.7\%) of abdominal pain. That is, side effects occurred in $11.9 \%$ of subjects. However these temporary occurrences were of mild severity and then improved spontaneously. The severity of these side effects, which are presumed to occur as a result of imipramine treatment, was not so great as to discontinue the administration of drugs.

In recent years, opinions explaining the prognostic indicators for treatment responses to drugs used for patients with $\mathrm{NE}$ have been controversial. In many aspects, however, they remain obscure. This is because etiological factors of NE have been proposed to include the changes of normal secretory pattern of anti-diuretic hormones, delayed maturation and physical development, psychopathological factors, environmental stress, instability of the urinary bladder, genetic factors, the disorder of awakening from sleeping and urinary tract infections [13].

Urine concentration ability is associated with the homeostasis of plasma sodium, and it is subject to various factors affecting the glomerulus and renal tubules including renin-angiotensin-aldosterone system, atrial natriuretic peptide, sympathetic nervous system and desmopressin/vasopressin [14]. According to Lackgren et al., following the classification as the responder group and the non-responder group for assessment of the treatment effect of desmopressin, the responder group showed an excellent degree of renal sensitivity to desmopressin, indicative of a decreased urine concentration in the kidney of the non-responder group [15]. According to Steffens 
et al., because urine osmolality is relatively lower in patients with $\mathrm{NE}$, its measurements are indicative of a depletion of arginine vasopressin within the plasma and this may lead to prediction of treatment response [13]. In our series, urine osmolality prior to treatment was measured as $461.2 \pm 192.7$ $\mathrm{mOsm} / \mathrm{kg}$ in the complete responder group and this was significantly the lowest value of the 3 groups. Compared with before treatment, urine osmolality following treatment was increased in all 3 groups. In the complete responder group, however, it was increased from $461.2 \pm 192.7 \mathrm{mOsm} / \mathrm{kg}$ to $591.2 \pm 159.8$ $\mathrm{mOsm} / \mathrm{kg}$. These results indicate that the incremental degree of urine osmolality following treatment was the highest in the complete responder group and this reached statistical significance. Therefore it can be inferred that there would be a good treatment response to concomitant treatment in the group where urine osmolality is measured as the lowest value prior to treatment and that there would be a good treatment response to concomitant treatment if a relatively greater incremental degree of urine osmolality is observed following treatment.

In addition, a better treatment response in older children and those with a lower frequency of $\mathrm{NE}$ was also reported [16], but this was not significant in our series. This study is limited by its descriptive nature, and by a lack of large-scale assessment of clinical outcomes. Therefore, further large-scale studies are warranted to establish criteria for accurate prediction of treatment responses.

Conclusively, in children with monosymptomatic NE, the treatment response to concomitant medication of imipramine with desmopressin was associated with changes in urine osmolality between before and after treatment. It can therefore be inferred that there would be a good treatment response in cases in which the degree of urine osmolality was relatively lower and its incremental degree following treatment was relatively higher.

\section{ACKNOWLEDGMENT}

This work was supported by a grant from the Chunma medical research foundation, Korea, 2014.

\section{CONFLICT OF INTEREST}

The authors declare no conflict of interest.

\section{REFERENCES}

1. Butler RJ. Establishment of working definitions in nocturnal enuresis. Arch Dis Child 1991;66:267-71.

2. Husmann DA. Enuresis. Urology 1996;48:184-93.

3. Forsythe WI, Redmond A. Enuresis and spontaneous cure rate. Study of 1129 enuretis. Arch Dis Child 1974;49:259-63.

4. Foxman B, Valdez RB, Brook RH. Childhood enuresis: prevalence, perceived impact, and prescribed treatments. Pediatrics 1986;77:482-7.

5. Kang JY, Ha IS, Chung HI, Kim KM. Therapeutic effects of imipramine and DDAVP in enuretic children: a randomized prospective study. Korean J Urol 2003;44:665-71. Korean.

6. Park YH, Yeo JH, Choi JY, Chung HS, Lee KS, Ko CW, et al. Efficacy and safety during the combination therapy of imipramine and desmopressin in primary monosymptomatic nocturnal enuresis. J Korean Soc Pediatr Nephrol 2004;8: 129-37. Korean.

7. Cho HM, Park JS, Lee JY. Can bladder capacity and urine specific gravity as well as other variables predict the responsiveness to desmopressin in enuretic children? Korean J Urol 2002;43:303-7. Korean.

8. MacLean RE. Imipramine hydrochloride (Tofranil) and enuresis. Am J Psychiatry 1960;117:551.

9. Hunsballe JM, Rittig S, Pedersen EB, Olesen OV, Djurhuus JC. Single dose imipramine reduces nocturnal urine output in patients with nocturnal enuresis and nocturnal polyuria. J Urol 1997;158:830-6.

10. Robson WL, Nørgaard JP, Leung AK. Hyponatremia in patients with nocturnal enuresis treated with DDAVP. Eur J Pediatr 1996;155:959-62.

11. Jung JY, Kim KM. Effects of combined drug therapy in the nocturnal enuretic patients. Korean J Urol 1998;39:70-6. Korean.

12. Reinberg $\mathrm{Y}$, vaughn $\mathrm{M}$. Treatment of refractory monosymptomatic nocturnal enuresis with combination therapy of desmopressin and imipramine. Pediatrics 1996;98(Suppl):8.

13. Steffens J, Netzer M, Isenberg E, Alloussi S, Ziegler M. Vasopressin deficiency in primary nocturnal enuresis. Results of a controlled prospective study. Eur Urol 1993;24:366-70.

14. Nevéus T, Läckgren G, Tuvemo T, Hetta J, Hjälmås $K$, Stenberg A. Enuresis--background and treatment. Scand J Urol Nephrol Suppl 2000;206:1-44.

15. Läckgren G, Nevéus T, Stenberg A. Diurnal plasma vasopressin and urinary output in adolescents with monosymptomatic nocturnal enuresis. Acta Paediatr 1997;86:385-90.

16. Rushton HG, Belman AB, Zaontz MR, Skoog SJ, Sihelnik $S$. The influence of small functional bladder capacity and other predictors on the response to desmopressin in the management of monosymptomatic nocturnal enuresis. J Urol 1996;156:651-5. 